International Mathematical Forum, 1, 2006, no. 39, 1943-1949

\title{
A note on the Dittert conjecture for permanents
}

\author{
Gi-Sang Cheon ${ }^{1}$ \\ Department of Mathematics \\ Sungkyunkwan University \\ Suwon 440-746, Rep. of Korea \\ Haeng-Won Yoon ${ }^{2}$ \\ Department of Mathematics \\ Daejin University \\ Pocheon 487-711, Rep. of Korea
}

\begin{abstract}
Let $K_{n}$ denote the convex set consisting of all real nonnegative $n \times n$ matrices whose entries have sum $n$. For $A \in K_{n}$ with row sums $r_{1}, \ldots, r_{n}$ and column sums $c_{1}, \ldots, c_{n}$, let $\psi$ be defined by

$$
\psi(A)=\prod_{i=1}^{n} r_{i}+\prod_{j=1}^{n} c_{j}-\operatorname{per}(A),
$$

where per stands for the permanent function. E. Dittert conjectures that the maximum of $\psi$ on $K_{n}$ occurs uniquely at $J_{n}=[1 / n]_{n \times n}$. The conjecture is still unsolved for $n \geq 4$. In this paper, we obtain some sufficient conditions for which the Dittert conjecture holds.
\end{abstract}

Keywords: Dittert conjecture, permanent.

Mathematics Subject Classification: 15A15.

\section{Introduction}

A square real nonnegative matrix is called row (resp. column) stochastic if all its row (resp. column) sums are equal to 1. A matrix which is both row

\footnotetext{
${ }^{1}$ The first author is partially supported by BK21 Project.

${ }^{2}$ The second author is partially supported by Daejin University during Sabbatical leave in 2006 .
} 
stochastic and column stochastic is called doubly stochastic. As usual, the set of all $n \times n$ doubly stochastic matrices is denoted by $\Omega_{n}$, and the $n \times n$ matrix all of whose entries equal $\frac{1}{n}$ is denoted by $J_{n}$.

For an $n \times n$ matrix $A=\left[a_{i j}\right]$, the permanent of $A$, $\operatorname{per}(A)$, is defined by

$$
\operatorname{per}(A)=\sum_{\pi \in S_{n}} a_{1 \pi(1)} \cdots a_{n \pi(n)}
$$

where $S_{n}$ denotes the permutations of $1, \ldots, n$ onto itself.

In 1926, van der Waerden [8] posed the problem of determining the minimum of the permanent function on $\Omega_{n}$.

It was conjectured that for any $A \in \Omega_{n}$,

$$
\operatorname{per}(A) \geq \frac{n !}{n^{n}}
$$

with equality holds if and only if $A=J_{n}$.

The conjecture remained unsolved for over half a century untill Egoryĉev [2] and Falikman [3] proved it independently. We will call this problem as the van der Waerden-Egoryĉev-Falikman Theorem.

In this paper, we consider a conjecture generalizing the van der WaerdenEgorycev-Falikman Theorem, which has known as the Dittert conjecture [6, Conjecture 28].

Throughout this paper, let $K_{n}$ denote the set of all real nonnegative $n \times n$ matrices whose entries have sum $n$, and let $\psi$ denote a real valued function on $K_{n}$ defined by

$$
\psi(A)=\prod_{i=1}^{n} \sum_{j=1}^{n} a_{i j}+\prod_{j=1}^{n} \sum_{i=1}^{n} a_{i j}-\operatorname{per}(A)
$$

for $A=\left[a_{i j}\right] \in K_{n}$. Since $K_{n}$ is a compact subset of a finite dimensional euclidean space, it contains a matrix $A$ such that $\psi(A) \geq \psi(X)$ for all $X \in K_{n}$. Such a matrix $A$ will be called a $\psi$-maximizing matrix on $K_{n}$.

The following conjecture due to E. Dittert is still open for $n \geq 4$.

DITTERT CONJECTURE For any $A \in K_{n}$,

$$
\psi(A) \leq 2-\frac{n !}{n^{n}}
$$

with equality holds if and only if $A=J_{n}$.

The Dittert Conjecture asserts that $J_{n}$ is the unique $\psi$-maximizing matrix on $K_{n}$. It is proved for $n=2$ [7] and for $n=3$ [4], and the other partial results 
for it are found in $[1,4,5,7]$. In particular, as noted in [5] (also see [7]), we see that if $A$ is a $\psi$-maximizing matrix on $K_{n}$ then

$$
0<\operatorname{per}(A) \leq \frac{n !}{n^{n}}
$$

Clearly, from the van der Waerden-Egoryĉev-Falikman Theorem, the Dittert Conjecture holds for a special subset $\Omega_{n}$ of $K_{n}$.

In this note, it is obtained a sufficient condition on $K_{n}$ for which the Dittert conjecture holds. As a consequence, we see that a $\psi$-maximizing matrix on $K_{n}$ depends on only its permanent.

\section{A sufficient condition for which the Dittert conjecture holds}

Throughout this section, let $A$ be a matrix on $K_{n}$ with row sums $r_{1}, \ldots, r_{n}$ and column sums $c_{1}, \ldots, c_{n}$ such that $r_{1}+\cdots+r_{n}=c_{1}+\cdots+c_{n}=n$. Then $\psi(A)$ in $(1)$ can be written as

$$
\psi(A)=\prod_{i=1}^{n} r_{i}+\prod_{j=1}^{n} c_{j}-\operatorname{per}(A) .
$$

Since $\operatorname{per}(A)=\operatorname{per}\left(A^{T}\right)$, it will enable us to replace "row" by "column" in all results of $\psi(A)$. Moreover, noticing for any $n \times n$ permutation matrices $P$ and $Q, \operatorname{per}(P A Q)=\operatorname{per}(A)$ and thus $\psi(P A Q)=\psi(A)$, without loss of generality we may assume that

$$
r_{1} \leq \cdots \leq r_{n} \text { and } c_{1} \leq \cdots \leq c_{n}
$$

Clearly, $r_{1} \leq 1$ and $c_{1} \leq 1$, and if $r_{1}=c_{1}=1$ then $A$ is a doubly stochastic matrix.

For a matrix $A \in K_{n}, A(i \mid j)$ denotes the $(n-1) \times(n-1)$ matrix obtained from $A$ by deleting the $i$-th row and the $j$-th column, and let

$$
\psi_{i j}(A)=\prod_{k \neq i} r_{k}+\prod_{l \neq j} c_{l}-\operatorname{per}(A(i \mid j)) .
$$

The following lemma is due to S. G. Hwang [5].

LEMMA 2.1 Let $A=\left[a_{i j}\right]$ be a $\psi$-maximizing matrix on $K_{n}$. Then

$$
\psi(A) \geq \psi_{i j}(A)
$$

with equality holds if $a_{i j}>0$. 
For $r_{i}>0$ and $c_{j}>0$, let

$$
r=\prod_{i=1}^{n} r_{i}, \quad c=\prod_{j=1}^{n} c_{j}, \quad \bar{r}_{i}=\frac{r}{r_{i}} \text { and } \bar{c}_{j}=\frac{c}{c_{j}} .
$$

THEOREM 2.2 Let $A=\left[a_{i j}\right]$ be a $\psi$-maximizing matrix on $K_{n}$. Then

$$
\psi(A)=\frac{1}{n^{2}} \sum_{i, j} \psi_{i j}(A) \quad \text { iff } \quad A=J_{n} .
$$

Proof. Clearly, if $A=J_{n}$ then $\psi(A)=\frac{1}{n^{2}} \sum_{i, j} \psi_{i, j}(A)$ holds. Suppose that $\psi(A)=\frac{1}{n^{2}} \sum_{i, j} \psi_{i j}(A)$. Lemma 2.1 implies that $\psi(A)=\psi_{i, j}(A)$ for all $i, j \in\{1,2, \ldots, n\}$. Noticing for any $i, s, t(s \neq t)$ in $\{1,2, \ldots, n\}$

$$
0=\psi_{i s}-\psi_{i t}=\bar{c}_{s}-\bar{c}_{t}+\operatorname{per} A(i \mid s)-\operatorname{per} A(i \mid t)
$$

we obtain

$$
\operatorname{per} A(i \mid s)-\operatorname{per} A(i \mid t)=\left(\frac{1}{c_{s}}-\frac{1}{c_{t}}\right) \prod_{j=1}^{n} c_{j} .
$$

By the (average) Lemma 2 [5], if $B$ is the matrix obtained from $A$ by averaging columns $s$ and $t$ then $\psi(A)=\psi(B)$. The similar result holds for rows. Consequently, after a sequence of averaging on columns and rows of $A$, we obtain $A=J_{n}$.

LEMMA 2.3 Let $A$ be a $\psi$-maximizing matrix on $K_{n}$ with (3). Then

$$
0<\operatorname{per}(A) \leq \operatorname{per}(A(1 \mid 1))
$$

with equality holds if $r_{s}=r_{t}$.

Proof. The $0<\operatorname{per}(A)$ follows from (2). By Lemma 2.1, we get

$$
\begin{aligned}
\psi(A)-\psi_{11}(A) & =r+c-\operatorname{per}(A)-\left(\bar{r}_{1}+\bar{c}_{1}-\operatorname{per}(A(1 \mid 1))\right. \\
& =\bar{r}_{1}\left(r_{1}-1\right)+\bar{c}_{1}\left(c_{1}-1\right)+\operatorname{per}(A(1 \mid 1))-\operatorname{per}(A) \geq 0,
\end{aligned}
$$

which implies that

$$
\bar{r}_{1}\left(r_{1}-1\right)+\bar{c}_{1}\left(c_{1}-1\right) \geq \operatorname{per}(A)-\operatorname{per}(A(1 \mid 1)) .
$$

Since $r_{1} \leq 1$ and $c_{1} \leq 1$ we have $\operatorname{per}(A) \leq \operatorname{per}(A(1 \mid 1))$, which completes the proof.

LEMMA 2.4 Let $A=\left[a_{i j}\right]$ be a $\psi$-maximizing matrix on $K_{n}$ with (3) and let $k$ be an integer such that $1 \leq k \leq n$. If $a_{s k}>0$ and $a_{t k}>0$ for any integers $s$ and $t$ such that $1 \leq s<t \leq n$, then

$$
\operatorname{per}(A(s \mid k)) \geq \operatorname{per}(A(t \mid k)),
$$


with equality holds if $r_{s}=r_{t}$.

Proof. By Lemma 2.1, we get

$$
\begin{aligned}
\psi_{s k}(A)-\psi_{t k}(A) & =\left(\bar{r}_{s}+\bar{c}_{k}-\operatorname{per}(A(s \mid k))-\left(\bar{r}_{t}+\bar{c}_{k}-\operatorname{per}(A(t \mid k))\right.\right. \\
& =\left(\frac{r}{r_{s}}-\frac{r}{r_{t}}\right)-(\operatorname{per}(A(s \mid k))-\operatorname{per}(A(t \mid k))) \\
& =\frac{r\left(r_{t}-r_{s}\right)}{r_{s} r_{t}}-(\operatorname{per}(A(s \mid k))-\operatorname{per}(A(t \mid k)))=0 .
\end{aligned}
$$

Since $r_{t} \geq r_{s}$, we have $\operatorname{per}(A(s \mid k)) \geq \operatorname{per}(A(t \mid k))$, and if $r_{s}=r_{t}$ then the equality holds. Thus the proof is completed.

The following is an immediate consequence of Lemma 2.4.

COROLLARY 2.5 Let $A=\left[a_{i j}\right]$ be a $\psi$-maximizing matrix on $K_{n}$ with (3) and let $k$ be an integer such that $1 \leq k \leq n$. If $a_{i k}>0$ for all $i=1,2, \ldots, n$, then

$$
\operatorname{per}(A(1 \mid k)) \geq \operatorname{per}(A(2 \mid k)) \geq \cdots \geq \operatorname{per}(A(n \mid k)),
$$

with all equalities hold if $A$ is a row stochastic matrix.

THEOREM 2.6 Let $A$ be a $\psi$-maximizing matrix on $K_{n}$ with (3). If

$$
\operatorname{per}(A)=\operatorname{per}(A(1 \mid 1))
$$

then the Dittert conjecture holds.

Proof. Note that $r_{1} \leq 1$ and $c_{1} \leq 1$. Suppose that $r_{1}<1$ or $c_{1}<1$. Then we get

$$
\begin{aligned}
\psi(A) & =r+c-\operatorname{per}(A) \\
& =r_{1} \cdot \bar{r}_{1}+c_{1} \cdot \bar{c}_{1}-\operatorname{per}(A(1 \mid 1))+\operatorname{per}(A(1 \mid 1))-\operatorname{per}(A) \\
& <\bar{r}_{1}+\bar{c}_{1}-\operatorname{per}(A(1 \mid 1))+\operatorname{per}(A(1 \mid 1))-\operatorname{per}(A) \\
& =\psi_{11}(A)+\operatorname{per}(A(1 \mid 1))-\operatorname{per}(A) .
\end{aligned}
$$

If $\operatorname{per}(A)=\operatorname{per}(A(1 \mid 1))$ then $\psi(A)<\psi_{11}(A)$, which contradicts to Lemma 2.1. Thus $r_{1}=1$ and $c_{1}=1$. It follows that $r_{1}=\cdots=r_{n}=1$ and $c_{1}=\cdots=c_{n}=1$. From the van der Waerden-Egorycev-Falikman Theorem we have $A=J_{n}$, and the proof is completed.

Now, we obtain a sufficient condition for which (4) holds. To prove our next theorem, we shall use Alexandrov's inequality for permanents:

$$
(\operatorname{per}(A))^{2} \geq \operatorname{per}\left[\mathrm{a}_{1}, \mathrm{a}_{1}, \mathrm{a}_{2}, \ldots, \mathrm{a}_{\mathrm{n}-1}\right] \operatorname{per}\left[\mathrm{a}_{2}, \ldots, \mathrm{a}_{\mathrm{n}-1}, \mathrm{a}_{\mathrm{n}}, \mathrm{a}_{\mathrm{n}}\right]
$$


for any nonnegative $n \times n$ matrix $A=\left[\mathrm{a}_{1}, \mathrm{a}_{2}, \ldots, \mathrm{a}_{\mathrm{n}}\right]$, which is a reformulation of the original one due to Egorycev [2], where $\mathrm{a}_{\mathrm{i}}$ is the $i$-th column vector of A.

THEOREM 2.7 Let $A=\left[a_{i j}\right]$ be a $\psi$-maximizing matrix on $K_{n}$ with (3). If $\operatorname{per}(A(1 \mid 1)) \leq \operatorname{per}(A(i \mid j))$ for all $i \in\{1, \ldots, n\}$ and $j \in\{1, n\}$, where $c_{1} c_{n} \geq 1$ then

$$
\operatorname{per}(A)=\operatorname{per}(A(1 \mid 1))
$$

Proof. From Lemma 2.3, suppose that $\operatorname{per}(A)<\operatorname{per}(A(1 \mid 1))$. Now Alexandrov's inequality (also see [2]) implies that

$$
\begin{aligned}
(\operatorname{per}(A))^{2} & \geq\left[\sum_{i=1}^{n} a_{i 1} \operatorname{per}(A(i \mid n))\right]\left[\sum_{i=1}^{n} a_{i n} \operatorname{per}(A(i \mid 1))\right] \\
& \geq\left(\sum_{i=1}^{n} a_{i 1}\right) \operatorname{per}(A(1 \mid 1))\left(\sum_{i=1}^{n} a_{i n}\right) \operatorname{per}(A(1 \mid 1)) \\
& =c_{1} c_{n}(\operatorname{per}(A(1 \mid 1)))^{2} \\
& >c_{1} c_{n}(\operatorname{per}(A))^{2}
\end{aligned}
$$

Since $c_{1} c_{n} \geq 1$ we have $(\operatorname{per}(A))^{2}>(\operatorname{per}(A))^{2}$, which is a contradiction. Hence $\operatorname{per}(A)=\operatorname{per}(A(1 \mid 1))$. This completes the proof.

\section{References}

[1] G.-S. Cheon and S.-G. Hwang, Maximization of a matrix function related to the Dittert conjecture, Linear Algebra Appl. 165 (1992), 153-165.

[2] G. P. Egoryĉev, A solution of van der Waerden's permanent problem, preprint IFSO-13M, Kirenski Inst. of Physics, Academy of Sciences of the USSR, Kransnojarsk, 1980.

[3] D. F. Falikman, A proof of van der Waerden's conjecture on the permanent of a stochastic matrix, Mat. Zametki 29 (1981), 931-938.

[4] S.-G. Hwang, On a conjecture of E. Dittert, Linear Algebra Appl. 95 (1987), 161-169.

[5] S.-G. Hwang, A note on a conjecture on permanents, Linear Algebra Appl. 71 (1986), 31-44.

[6] H. Minc, Theory of permanents 1982-1985, Linear and Multilinear Algebra 21 (1987), 109-148. 
[7] R. Sinkhorn, A problem related to the van der Waerden permanent theorem, Linear and Multilinear Algebra 16 (1984), 167-173.

[8] B. L. van der Waerden, Aufgabe 45, Jahresber Deutch Math.-Verein. 35 (1926), 117.

Received: April 12, 2006 